\title{
Phenomenological study of the electronic transport coefficients of graphene
}

\author{
N. M. R. Peres ${ }^{1}$, J. M. B. Lopes dos Santos ${ }^{2}$, and T. Stauber ${ }^{3}$, \\ ${ }^{1}$ Center of Physics and Department of Physics, University of Minho, P-4710-057, Braga, Portugal \\ ${ }^{2}$ CFP and Departamento de Física, Faculdade de Ciências Universidade de Porto, 4169-007 Porto, Portugal and \\ ${ }^{3}$ Instituto de Ciencia de Materiales de Madrid. CSIC. Cantoblanco. E-28049 Madrid, Spain
}

(Dated: October 24, 2018)

\begin{abstract}
Using a semi-classical approach and input from experiments on the conductivity of graphene, we determine the electronic density dependence of the electronic transport coefficients - conductivity, thermal conductivity and thermopower - of doped graphene. Also the electronic density dependence of the optical conductivity is obtained. Finally we show that the classical Hall effect (low field) in graphene has the same form as for the independent electron case, characterized by a parabolic dispersion, as long as the relaxation time is proportional to the momentum.
\end{abstract}

PACS numbers: 72.10.-d, 72.15.Jf, 72.15.Lh, 65.40.-b

Introduction. Since the experimental measurement of an electric field effect in graphene $\mathrm{e}^{1}$ and the observation of the odd integer quantum Hall effect 2,3 the electronic properties of graphene have been attracting a considerable attention from the community. Recent qualitative reviews $4,5,6$ on the physics of graphene give a brief account on both the experimental and theoretical status of the field.

The measured conductivity of graphene has two distinct fingerprints: a linear dependence upon the gate voltage of the DC conductivity, and a minimum conductivity at the neutrality point of one electron per carbon atom. The value of the conductivity minimum is of the order of the quantum of conductance $e^{2} / h$. This minimum of conductivity is a quantum mechanical effect which comes about as a consequence of disorder ${ }^{7}$. Disorder promotes a finite density of states at the Dirac point which is responsible for the minimum of conductivity, $\sigma_{\min }$. Unfortunately there are a number of different theoretical predictions available for $\sigma_{\min }$ in the literature $8.9,10,11,12,13,14,15,16$. Except for the numerical work of Nomura and MacDonald $\frac{17}{}$ all the calculations for the conductivity minimum are off by some numerical factor from the experimentally measured value.

Also interesting by itself is the linear dependence of the conductivity on the gate potential. Since the gate potential depends linearly on the electronic density, $n$, one has a conductivity $\sigma \propto n$. As shown by Shon and Ando $\underline{18}$ if the scatterers are short range one obtains a DC conductivity that is independent of the electronic density, at odds with the experimental result. Nomura and MacDonald ${ }^{17,19}$ showed that considering a scattering mechanics based on screened charged impurities it is possible to obtain from a Boltzmann equation approach a conductivity varying linearly with the density, in agreement with the experimental result. In this brief report we address the problem of finding the electronic density dependence of several transport properties of graphene. Since the electronic density is easily controlled by a gate voltage the expressions we derive here can certainly be tested experimentally. We therefore present in what follows a calculation of the electronic density dependences of the DC conductivity, the thermal conductivity and the thermopower. In addition, we study how the electronic density enters in the optical conductivity. Finally, we show that a certain form of the scattering rate is necessary if the classical expression for the Hall effect is to be maintained, in agreement with the experiments. A similar semi-classical approach was used by Falkovsky 20 to study the temperature dependence of the Hall conductivity in graphene. The link between the Kubo-Streda formulation and the semi-classical Boltzmann equation approach was discussed, in the context of massive Dirac fermions, by Sinitsyn et al. $\underline{21}$

Boltzmann equation and relaxation times. The Boltzmann equation has the form ${ }^{22}$

$$
-\boldsymbol{v}_{\boldsymbol{k}} \cdot \nabla_{\boldsymbol{r}} f\left(\epsilon_{\boldsymbol{k}}\right)-e / \hbar\left(\boldsymbol{E}+\boldsymbol{v}_{\boldsymbol{k}} \times H\right) \cdot \nabla_{\boldsymbol{k}} f\left(\epsilon_{\boldsymbol{k}}\right)=-\left.\frac{\partial f_{\boldsymbol{k}}}{\partial t}\right|_{\text {scatt } .} .
$$

The solution of the Boltzmann equation in its general form is difficult and one needs therefore to rely upon some approximation. The first step in the usual approximation scheme is to write the distribution as $f\left(\epsilon_{\boldsymbol{k}}\right)=$ $f^{0}\left(\epsilon_{\boldsymbol{k}}\right)+g\left(\epsilon_{\boldsymbol{k}}\right)$ where $f^{0}\left(\epsilon_{\boldsymbol{k}}\right)$ is the steady state distribution function and $g\left(\epsilon_{\boldsymbol{k}}\right)$ is assumed to be small. Inserting this ansatz in Eq. (1) and keeping only terms that are linear in the external fields one obtains the linearized Boltzmann equation ${ }^{22}$ which reads

$$
\begin{aligned}
& -\frac{\partial f^{0}\left(\epsilon_{\boldsymbol{k}}\right)}{\partial \epsilon_{\boldsymbol{k}}} \boldsymbol{v}_{\boldsymbol{k}} \cdot\left[\left(-\frac{\epsilon_{\boldsymbol{k}}-\zeta}{T}\right) \boldsymbol{\nabla}_{\boldsymbol{r}} T+e\left(\boldsymbol{E}-\frac{1}{e} \boldsymbol{\nabla}_{\boldsymbol{r}} \zeta\right)\right]= \\
& -\left.\frac{\partial f_{\boldsymbol{k}}}{\partial t}\right|_{\text {scatt. }}+\boldsymbol{v}_{\boldsymbol{k}} \cdot \boldsymbol{\nabla}_{\boldsymbol{r}} g_{\boldsymbol{k}}+\frac{e}{\hbar}\left(\boldsymbol{v}_{\boldsymbol{k}} \times \boldsymbol{H}\right) \cdot \boldsymbol{\nabla}_{\boldsymbol{k}} g_{\boldsymbol{k}} .
\end{aligned}
$$

The second approximation has to do with the form of the scattering term. The simplest approach is to introduce a relaxation time into the formalism. This is done by considering the approximation

$$
-\left.\frac{\partial f_{k}}{\partial t}\right|_{s c a t t .} \rightarrow \frac{g_{k}}{\tau_{k}}
$$

where $\tau_{\boldsymbol{k}}$ is the relaxation time, assumed to be momentum dependent. Its momentum dependence will be de- 


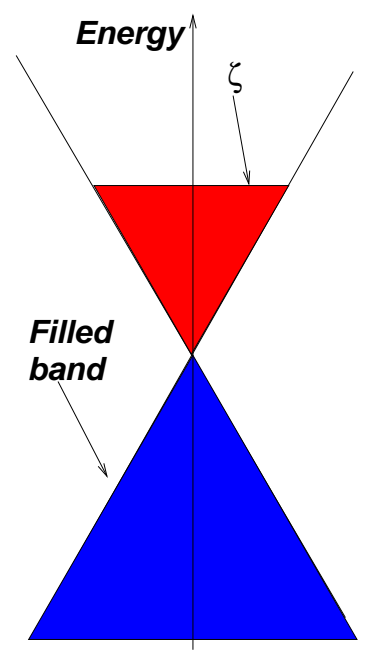

FIG. 1: (Color online) Dirac cones in graphene, in a situation of finite electronic density. The valence band is inert as long as the energy excitation processes are smaller than $\zeta$ (the Fermi energy).

termined phenomenologically in such way that the dependence of the conductivity upon the electronic density agrees with the experimental data. The Boltzmann equation is certainly not valid at the Dirac point, but since many experiments are done at finite carrier density, controlled by an external gate voltage, we expect the Boltzmann equation to give reliable results when an appropriate form for $\tau_{\boldsymbol{k}}$ is used.

Let us compute the Boltzmann relaxation time, $\tau_{\boldsymbol{k}}$, for two different scattering potentials:(i) a delta function potential; (ii) a screened Coulomb potential. The relaxation time $\tau_{\boldsymbol{k}}$ is defined as ( $\boldsymbol{k}$ is the momentum)

$$
\frac{1}{\tau_{\boldsymbol{k}}}=N_{i} A \int d \theta \int \frac{k^{\prime} d k^{\prime}}{(2 \pi)^{2}} S\left(\boldsymbol{k}, \boldsymbol{k}^{\prime}\right)(1-\cos \theta),
$$

where $N_{i}$ is the total number of impurities in the sample, $A$ is the area of the graphene sheet, and the transition rate $S\left(\boldsymbol{k}, \boldsymbol{k}^{\prime}\right)$ is given by

$$
S\left(\boldsymbol{k}, \boldsymbol{k}^{\prime}\right)=\frac{2 \pi}{\hbar}\left|H_{\boldsymbol{k}^{\prime}, \boldsymbol{k}}\right|^{2} \frac{1}{v_{F} \hbar} \delta\left(k^{\prime}-k\right),
$$

where the $v_{F} \hbar k$ is the dispersion of Dirac fermions in graphene and $H_{\boldsymbol{k}^{\prime}, \boldsymbol{k}}$ is defined as

$$
H_{\boldsymbol{k}^{\prime}, \boldsymbol{k}}=\int d^{2} r \psi_{\boldsymbol{k}^{\prime}}^{*}(\boldsymbol{r}) U_{S}(\boldsymbol{r}) \psi_{\boldsymbol{k}}(\boldsymbol{r})
$$

with $U_{S}(\mathrm{r})$ the scattering potential and $\psi_{\boldsymbol{k}}(\boldsymbol{r})$ is the electronic spinor wave function of a clean graphene sheet. If the potential is short range, $\frac{18}{,}$ of the form $U_{S}=v_{0} \delta(\boldsymbol{r})$, the Boltzmann relaxation time turns out to be

$$
\tau_{k}=\frac{4 \hbar^{2} v_{F}}{n_{i} v_{0}^{2}} \frac{1}{k}
$$

where $n_{i}$ is the impurity concentration per unit area. On the other hand, if the potential is the screened Coulomb potential, given by $U_{S}(\boldsymbol{r})=e Q e^{-r / L_{D}} /\left(4 \pi \epsilon_{0} \epsilon r\right)$ for charged impurities of charge $Q$, the relaxation time is given by

$$
\frac{1}{\tau_{\boldsymbol{k}}}=\frac{u_{0}^{2}}{v_{F} \hbar^{2} k}\left(1-\frac{\sqrt{1+4 k^{2} L_{D}^{2}}-1}{2 k^{2} L_{D}^{2}}\right),
$$

where $u_{0}^{2}=n_{i} Q^{2} e^{2} /\left(16 \epsilon_{0}^{2} \epsilon^{2}\right)$. In the limit $L_{D} \rightarrow \infty$ one obtains

$$
\tau_{k}=\frac{v_{F} \hbar^{2}}{u_{0}^{2}} k
$$

As we argue below, the phenomenology of Dirac fermions implies that the scattering in graphene must be of the form (9). In what follows we explore the consequences of this type of relaxation time.

At zero temperature the chemical potential $\zeta$ (Fermi energy) is related to the density of charge carriers (see Fig. (1) in the conduction band by $n=\pi^{-1}\left(\zeta / v_{F} \hbar\right)^{2}$. In what follows we give the expressions for the transport coefficients in terms of $\zeta$.

The DC conductivity. Within the relaxation time approximation the solution of the linearized Boltzmann equation when an electric field is applied to the sample is

$$
g_{\boldsymbol{k}}=-\frac{\partial f^{0}\left(\epsilon_{\boldsymbol{k}}\right)}{\partial \epsilon_{\boldsymbol{k}}} e \tau_{\boldsymbol{k}} \boldsymbol{v}_{\boldsymbol{k}} \cdot \boldsymbol{E}
$$

and the electric current reads

$$
\boldsymbol{J}=\frac{4}{A} \sum_{k} e \boldsymbol{v}_{\boldsymbol{k}} g_{\boldsymbol{k}}
$$

Since at low temperature the following relation $-f^{0}\left(\epsilon_{\boldsymbol{k}}\right) / \partial \epsilon_{\boldsymbol{k}} \rightarrow \delta\left(\zeta-v_{F} \hbar k\right)$ holds, one can easily see that assuming Eq. (9) where $k$ is measured relatively to the Dirac point, the electronic conductivity turns out to be

$$
\sigma_{x x}=2 \frac{e^{2}}{h} \frac{\zeta^{2}}{u_{0}^{2}}=2 \frac{e^{2}}{h} \frac{\pi\left(\hbar v_{F}\right)^{2}}{u_{0}^{2}} n,
$$

where $u_{0}$ is the strength of the scattering potential (with dimensions of energy). The electronic conductivity depends linearly on the particle density, in agreement with the experimental data $\stackrel{1,2}{=}$ and as was first noted by Nomura and MacDonald in Ref $\underset{19}{\underline{19}}$. The form used for $\tau_{\boldsymbol{k}}$ is thus imposed both by dimensional analysis and by phenomenology. We note that for short range scatterers 17,18 $\tau_{\boldsymbol{k}} \propto k^{-1}$, leading to an DC conductivity that is independent of the density and therefore in disagreement with the experiments.

Having settled the need for Eq. (9) we now proceed to determine the density dependence of the other transport coefficients. We stress that the Coulomb potential is one possible mechanism of producing a scattering rate of the 
form (9) but we do not exclude that other mechanisms may exist.

The optical conductivity. Here we want to obtain the electronic density dependence of the optical conductivity of a doped graphene plane. Since the Boltzmann approach does not include inter-band transitions, the expressions obtained below are only valid as long as $\hbar \omega \leq \zeta$, where the above mentioned transitions are blocked by the Pauli principle.

Our aim is to obtain the response of the electronic system to an external electric field of the form

$$
\boldsymbol{E}=\boldsymbol{E}_{0} e^{i(\boldsymbol{q} \cdot \boldsymbol{r}-\omega t)} .
$$

The Boltzmann equation has, for this problem, the form

$$
-\frac{\partial f^{0}\left(\epsilon_{\boldsymbol{k}}\right)}{\partial \epsilon_{\boldsymbol{k}}} e \boldsymbol{v}_{\boldsymbol{k}} \cdot \boldsymbol{E}=\frac{g_{\boldsymbol{k}}}{\tau_{\boldsymbol{k}}}+\boldsymbol{v}_{\boldsymbol{k}} \cdot \nabla_{\boldsymbol{r}} g_{\boldsymbol{k}}+\frac{\partial g_{\boldsymbol{k}}}{\partial t}
$$

The solution of the linearized Boltzmann equation (14) is well known 22 , reading

$$
g_{\boldsymbol{k}}=-\frac{\partial f^{0}\left(\epsilon_{\boldsymbol{k}}\right)}{\partial \epsilon_{\boldsymbol{k}}} \Phi_{\boldsymbol{q}}(\omega, \boldsymbol{k}) e^{i(\boldsymbol{q} \cdot \boldsymbol{r}-\omega t)},
$$

with

$$
\Phi_{\boldsymbol{q}}(\omega, \boldsymbol{k})=\frac{e \tau_{\boldsymbol{k}} \boldsymbol{v}_{\boldsymbol{k}} \cdot \boldsymbol{E}_{0}}{1-i \omega \tau_{\boldsymbol{k}}+i \tau_{\boldsymbol{k}} \boldsymbol{q} \cdot \boldsymbol{v}_{\boldsymbol{k}}}
$$

The Fourier component, $\boldsymbol{J}(\omega, \boldsymbol{q})$, of the current is given by

$$
\boldsymbol{J}(\omega, \boldsymbol{q})=\frac{1}{\pi^{2}} \int d^{2} k e \boldsymbol{v}_{\boldsymbol{k}} \Phi_{\boldsymbol{q}}(\omega, \boldsymbol{k})\left(-\frac{\partial f^{0}\left(\epsilon_{\boldsymbol{k}}\right)}{\partial \epsilon_{\boldsymbol{k}}}\right),
$$

leading in the long-wavelength limit to an optical conductivity of the form

$$
\sigma_{x x}(\omega)=2 \frac{e^{2}}{h} \frac{\zeta^{2}}{u_{0}^{2}} \frac{1+i \omega \hbar \zeta / u_{0}^{2}}{1+\left(\omega \hbar \zeta / u_{0}^{2}\right)^{2}} .
$$

What should be stressed about Eq. (18) is its density dependence. Since the electronic density is proportional to $\zeta^{2}$ it should be possible to collapse the optical conductivity curves for different densities in a representation of $\sigma_{x x}(\omega) / n$ versus $\omega \sqrt{n}$. This prediction can easily be confirmed by reflectance measurements in graphene, for frequencies below $\zeta$. Moreover, since the electronic density induced by a gate voltage $V_{g}$ is proportional to the gate voltage, by the relation $n=\frac{\epsilon \epsilon_{0}}{t e} V_{g}$, where $t$ is the thickness of the Silicon oxide substrate, one obtains a collapse of the optical conductivity for different gate voltages, by representing $\sigma_{x x}(\omega) / V_{g}$ versus $\omega \sqrt{V}_{g}$.

Thermal conductivity and thermopower. Our next goal is to obtain the density dependence of the thermal conductivity and of the thermopower. For a discussion within the Kubo-formalism, see Ref. 23 .

In the presence of a temperature gradient in the sample, the linearized Boltzmann equation has the form

$$
-\frac{\partial f^{0}\left(\epsilon_{\boldsymbol{k}}\right)}{\partial \epsilon_{\boldsymbol{k}}} \boldsymbol{v}_{\boldsymbol{k}} \cdot\left[\left(-\frac{\epsilon_{\boldsymbol{k}}-\zeta}{T}\right) \boldsymbol{\nabla}_{\boldsymbol{r}} T+e \boldsymbol{E}_{o b s v .}\right]=\frac{g_{\boldsymbol{k}}}{\tau_{\boldsymbol{k}}}
$$

where the measured electric field is given by $\boldsymbol{E}_{\text {obsv. }}=$ $\boldsymbol{E}-\nabla_{\boldsymbol{r}} \zeta / e$. In this situation we have, in addition to the electric current, a heat current (flux of heat per unit of area) given by

$$
\boldsymbol{U}=\frac{4}{A} \sum_{\boldsymbol{k}} \boldsymbol{v}_{\boldsymbol{k}}\left(\epsilon_{\boldsymbol{k}}-\zeta\right) g_{\boldsymbol{k}}
$$

Both the electric and the heat currents can be written $\operatorname{as}^{22}$

$$
\begin{aligned}
\boldsymbol{J} & =e^{2} \boldsymbol{K}_{0} \cdot \boldsymbol{E}_{\text {obsv }}+\frac{e}{T} \boldsymbol{K}_{1} \cdot\left(-\nabla_{\boldsymbol{r}} T\right) \\
\boldsymbol{U} & =e \boldsymbol{K}_{1} \cdot \boldsymbol{E}_{\text {obsv. }}+\frac{1}{T} \boldsymbol{K}_{2} \cdot\left(-\boldsymbol{\nabla}_{\boldsymbol{r}} T\right),
\end{aligned}
$$

where $\boldsymbol{K}_{i}, i=0,1,2$ are second order tensors. In this problem the tensors are diagonal, i.e. $\boldsymbol{K}_{i}=\mathbf{1} k_{i}$, and by a well established procedure $\frac{22}{2}$ one obtains

$$
\begin{aligned}
k_{0} & =\frac{2}{h} \frac{\zeta^{2}}{u_{0}^{2}}, \\
k_{1} & =\frac{4}{3} \frac{\pi^{2}}{h}\left(k_{B} T\right)^{2} \frac{\zeta}{u_{0}^{2}}, \\
k_{2} & =\frac{2}{3} \frac{\pi^{2}}{h}\left(k_{B} T\right)^{2} \frac{\zeta^{2}}{u_{0}^{2}} .
\end{aligned}
$$

From the results (22), (23) and (24) it is easy to derive both the thermal conductivity $\kappa$ and the thermopower $Q$. These are given by

$$
\kappa=\frac{1}{T}\left(\frac{2}{3} \frac{\pi^{2}}{h}\left(k_{B} T\right)^{2} \frac{\zeta^{2}}{u_{0}^{2}}-\frac{8}{9} \frac{\pi^{4}}{h}\left(k_{B} T\right)^{4} \frac{1}{u_{0}^{2}}\right)
$$

and

$$
Q=\frac{1}{e T} \frac{2}{3} \frac{\pi^{2}}{\zeta}\left(k_{B} T\right)^{2}
$$

Again, what should be emphasized in these results is the dependence of both $\kappa$ and $Q$ on the particle density, which is different from that of the usual two dimensional electron gas. Since it is experimentally feasible to control the carrier density in the graphene plane ${ }^{1}$ it is possible to check experimentally the dependence of the transport coefficients on the particle density.

Hall effect. In what follows we prove that the scattering rate (9) leads to a classical (low field) Hall coefficient $R$ given by

$$
R=\frac{1}{e n}
$$

in agreement with the experiments. For a discussion within the Kubo-formalism, see Ref. ${ }^{24}$.

The linearized Boltzmann equation in the presence of a static magnetic field, perpendicular to the graphene plane $H=(0,0, B)$, is given by

$$
\left(-\frac{\partial f\left(\epsilon_{\boldsymbol{k}}\right)}{\partial \epsilon_{\boldsymbol{k}}}\right) e \boldsymbol{E} \cdot \boldsymbol{v}_{\boldsymbol{k}}=\frac{g_{\boldsymbol{k}}}{\tau_{\boldsymbol{k}}}+\frac{e}{\hbar}\left(\boldsymbol{v}_{\boldsymbol{k}} \times \boldsymbol{H}\right) \cdot \nabla_{\boldsymbol{k}} g_{\boldsymbol{k}} .
$$


The determination of $g_{k}$ becomes quite simple by realizing that the right-hand-side of Eq. (28) (after multiplication by $\tau_{\boldsymbol{k}}$ ) is the first order expansion of

$$
g_{\boldsymbol{k}+\tau_{\boldsymbol{k}}(e / \hbar)\left(\boldsymbol{v}_{\boldsymbol{k}} \times \boldsymbol{H}\right)} .
$$

Since $\boldsymbol{v}_{\boldsymbol{k}}=v_{F} \boldsymbol{k} / k$ and $\tau_{\boldsymbol{k}}$ is given by Eq. (9), Eq. (29) can be rewritten as $g_{\boldsymbol{k}+\left(\alpha e v_{F} / \hbar\right)(\boldsymbol{k} \times \boldsymbol{H})}$, where $\alpha=$ $v_{F} \hbar^{2} u_{0}^{-2}$. We now define a new momentum variable $\boldsymbol{k}^{\prime}$ given by

$$
\boldsymbol{k}^{\prime}=\boldsymbol{k}+\frac{\alpha e v_{F}}{\hbar}(\boldsymbol{k} \times \boldsymbol{H}) .
$$

Solving Eq. (30) for $\boldsymbol{k}$ as function of $\boldsymbol{k}^{\prime}$ and making the left hand side of Eq. (28) equal to $g_{\boldsymbol{k}^{\prime}}$, one obtains the solution

$g(\boldsymbol{k})=\left(-\frac{\partial f\left(\epsilon_{\boldsymbol{k}}\right)}{\partial \epsilon_{\boldsymbol{k}}}\right) e v_{F} \alpha \boldsymbol{k} \cdot\left(\boldsymbol{E}-\alpha e v_{F} \boldsymbol{H} \times \boldsymbol{E} / \hbar\right) /\left(1+a^{2}\right)$,

with $a=v_{F}^{2} \hbar^{2} /\left(l_{B}^{2} u_{0}^{2}\right)$ and $l_{B}^{2}=\hbar /(e B)$.

Knowing the solution to $g(\boldsymbol{k})$ the electric current is easily computed giving

$$
\boldsymbol{J}=\frac{\sigma_{x x}}{1+a^{2}}(\boldsymbol{E}-a \hat{z} \times \boldsymbol{E})
$$

where $\hat{z}$ is a unit vector along the $z$ direction. The conductivity tensor $\boldsymbol{\sigma}$ is therefore given by

$$
\boldsymbol{\sigma}=\frac{\sigma_{x x}}{1+a^{2}}\left(\begin{array}{cc}
1 & a \\
-a & 1
\end{array}\right)
$$

In the traditional Hall set up one has $J_{y}=0$ leading to

$$
J_{x}=\sigma_{x x} E_{x}
$$

meaning that there is no magnetoresistance, and

$$
E_{y}=\frac{\pi v_{F}^{2} \hbar^{2}}{\zeta^{2} e} H J_{x}=\frac{1}{n e} H J_{x}
$$

which produces a $R$ Hall constant given by Eq. (27). This result is in agreement with the experimental findings in the low field Hall effect 1,2 .

Conclusions. In this brief report we derived, using a semi-classical approach, the electronic density dependence of the DC conductivity, the optical conductivity, the thermal conductivity, the thermopower and the classical (low field) Hall effect. Our proposed expressions are based on a phenomenological equation for the scattering rate of the Dirac electrons. Some of our findings have already received experimental confirmation; the results for the thermal properties and for the optical conductivity can be tested experimentally. The Boltzmann approach can not explain the universal conductivity value occurring for $n \rightarrow 0$, that is, our approach breaks down at the neutrality point.

Acknowledgments: The authors want to thank F. Guinea, Shan-Wen Tsai, and A. H. Castro Neto for useful comments and many discussions. This work has been supported by MEC (Spain) through Grant No. FIS200406490-C03-00, by the European Union, through contract 12881 (NEST), and the Juan de la Cierva Program (MEC, Spain). N. M. R. P. and J. M. B. L. S. thank FCT under the grant PTDC/FIS/64404/2006.
1 K. S. Novoselov, A. K. Geim, S. V. Morozov, D. Jiang, Y. Zhang, S. V. Dubonos, I. V. Grigorieva, and A. A. Firsov, Science 306, 666 (2004).

${ }^{2}$ K. S. Novoselov, A. K. Geim, S. V. Morozov, D. Jiang, M. I. Katsnelson, I. V. Grigorieva, S. V. Dubonos, and A. A. Firsov, Nature 438, 197 (2005).

3 Y. Zhang, Y.-W. Tan, H. L. Stormer, and P. Kim, Nature 438, 201 (2005).

4 A. K. Geim and K. S. Novoselov, Nature Materials 6, 183 (2007).

${ }^{5}$ M. I. Katsnelson, Materials Today 10, 20 (2007).

6 A. H. Castro Neto, F. Guinea, N. M. R. Peres, Physics World, November, 33 (2006).

7 N. M. R. Peres, F. Guinea, and A. H. Castro Neto, Phys. Rev. B 73, 125411 (2006).

${ }^{8}$ K. Ziegler, cond-mat/0701300

9 A.W.W. Ludwig et al., Phys. Rev. B 50, 7526 (1994).

10 K. Ziegler, Phys. Rev. B 55, 10661 (1997); Phys. Rev. Lett. 80, 3113 (1998).

11 M.I. Katsnelson, Eur. Phys. J. B 51, 157 (2006).

12 V.P. Gusynin and S.G. Sharapov, Phys. Rev. Lett. 95, 146801 (2005).
13 J. Tworzydlo, B. Trauzettel, M. Titov, A. Rycerz, and C.W.J. Beenakker, Phys. Rev. Lett. 96, 246802 (2006).

14 L.A. Falkovsky and A.A. Varlamov, cond-mat/0606800

15 J. Cserti, cond-mat/0608219

16 K. Ziegler, Phys. Rev. Lett. 97, 266802 (2006).

17 Kentaro Nomura and A.H. MacDonald, Phys. Rev. Lett. 98, 076602 (2007).

18 N. H. Shon and T. Ando, J. Phys. Soc. Jpn. 67, 2421 (1998).

19 Kentaro Nomura and A.H. MacDonald, Phys. Rev. Lett. . 96, 256602 (2006).

20 L. A. Falkovsky, Phys. Rev. B 75, 033409 (2007).

21 N. A. Sinitsyn, A. H. MacDonald, T. Jungwirth, V. K. Dugaev, and Jairo Sinova, Phys. Rev. B 75045315 (2007).

22 J. M. Ziman, Principles of the Theory of Solids, $2^{\text {nd }}$ Edition, (Cambridge, 1972).

23 V. P. Gusynin and S. G. Sharapov, Phys. Rev. B 73, 245411 (2006).

24 S. G. Sharapov, V. P. Gusynin, and H. Beck, Phys. Rev. B 67, 144509 (2003). 